\title{
On a fractional Henon equation and applications
}

\author{
YANNICK SiRE AND Jun-Cheng WeI
}

\begin{abstract}
We investigate some nonlocal equations in bounded domains for different kinds of operators with power nonlinearities. We prove an existence result in the spirit of an earlier work by W.-M. Ni. As an application, we prove the existence of solutions to nonlocal supercritical equations in the complement of a ball. We prove also some regularity results for higher order nonlocal equations, of independent interest.
\end{abstract}

\section{Introduction}

This paper is devoted to a fractional version of the Henon equation and some applications. As far as the existence result is concerned, we consider two types of operators: the one defined spectrally on the unit ball $B_{1} \subset \mathbb{R}^{n}$ and the one defined on the whole of $\mathbb{R}^{n}$ but restricted to functions defined on the bounded domain $B_{1}$ and vanishing outside $B_{1}$. One feature of the present paper is to consider any powers between 0 and $n / 2$. As far as we know, this is rather new in the field and introduces several new difficulties.

\section{The operators}

In this section, we define the two operators under consideration.

- The spectral Laplacian: We first define the operator $A_{s}$ as described for instance in [5]. Let $\left\{\varphi_{k}\right\}_{k=1}^{\infty}$ be an orthonormal basis of $L^{2}\left(B_{1}\right)$ consisting of eigenfunctions of $-\Delta$ in $B_{1}$ with homogeneous Dirichlet boundary conditions, associated to the eigenvalues $\left\{\lambda_{k}\right\}_{k=1}^{\infty}$. Namely, $0<\lambda_{1}<\lambda_{2} \leq \lambda_{3} \leq$

The research of YS is partially supported by ANR "HAB" and ERC "EPSILON".

The research of JW is partially supported by NSERC of Canada. 
$\cdots \leq \lambda_{k} \rightarrow+\infty, \int_{B_{1}} \varphi_{j} \varphi_{k} d x=\delta_{j, k}$ and

$$
\begin{cases}-\Delta \varphi_{k}=\lambda_{k} \varphi_{k} & \text { in } B_{1} \\ \varphi_{k}=0 & \text { on } \partial B_{1}\end{cases}
$$

The operator $A_{s}$ is defined for any $u \in C_{c}^{\infty}\left(B_{1}\right)$ by

$$
A_{s} u=\sum_{k=1}^{\infty} \lambda_{k}^{s} u_{k} \varphi_{k}
$$

where

$$
u=\sum_{k=1}^{\infty} u_{k} \varphi_{k}, \quad \text { and } \quad u_{k}=\int_{B_{1}} u \varphi_{k} d x .
$$

This operator can be extended by density for $u$ in the Hilbert space

$$
\mathcal{H}^{s}\left(B_{1}\right)=\left\{u \in L^{2}\left(B_{1}\right): \sum_{k=1}^{\infty} \lambda_{k}^{s}\left|u_{k}\right|^{2}<+\infty\right\} .
$$

Note that the operator $A_{s}$ realizes an isomorphism between $\mathcal{H}^{s}$ and its dual. It happens that the space $\mathcal{H}^{s}\left(B_{1}\right)$ can be fully characterized as it has been done in $[2,5]$

- The restricted Laplacian: The second operator we consider is the classical fractional Laplacian $(-\Delta)^{s}$ defined on all of $\mathbb{R}^{n}$. This is a Fourier multiplier of symbol $|\xi|^{2 s}$ in $\mathcal{S}^{\prime}\left(\mathbb{R}^{n}\right)$, the space of tempered distributions. One can define this operator by using the integral representation in terms of hypersingular kernels

$$
\left(-\Delta_{\mathbb{R}^{n}}\right)^{s} u(x)=c_{d, s} \text { P.V. } \int_{\mathbb{R}^{n}} \frac{u(x)-u(z)}{|x-z|^{n+2 s}} d z,
$$

where $c_{d, s}>0$ is a normalization constant. In this case we materialize the zero Dirichlet condition by restricting the operator to act only on functions that are zero outside $B_{1}$. We will denote this operator $\left(-\left.\Delta\right|_{\Omega}\right)^{s}$.

Remark 1.1. Note that both operators are different by many ways even for powers $s \in(0,1)$ as described extensively in [2] (see also [11] for an explanation why the spectra of each operators are different).

\section{Problems under consideration}

We denote $[s]$ the fractional part of $s$ so that $s=m+[s]$ where $m \in \mathbb{N}$. If $s \in(0,1)$, clearly we have $m=0$. We consider first the following problem 
$n \geq 2$

$$
\left\{\begin{array}{l}
A_{s} u=|x|^{\alpha} u^{p} \text { in } B_{1}, \\
u=\Delta u=. .=\Delta^{m-1} u=0 \text { on } \partial B_{1},
\end{array}\right.
$$

with the convention that if $s \in(0,1)$, then the only remaining boundary condition on $\partial B_{1}$ is $u=0$.

We will also consider the following problem

$$
\left\{\begin{array}{l}
(-\Delta)^{s} u=|x|^{\alpha} u^{p} \text { in } B_{1} \\
u=0 \text { in } B_{1}^{c}
\end{array}\right.
$$

Note that here one do not need to assume higher order boundary conditions.

In both cases, we assume that $p>1$ and $\alpha>0$. We will be more precise later for the allowed ranges of the power $s$.

We finally finish this section with the definition of weak solutions for our problems. We have

Definition 1.2. A solution $u \in \mathcal{H}^{s} \cap L^{p}\left(B_{1}\right)$ such that $u=\Delta u=\cdots=$ $\Delta^{m-1} u=0$ on $\partial B_{1}$ is a weak solution of problem (1.4) if for any $\varphi \in C_{0}^{\infty}\left(B_{1}\right)$ one has

$$
\int_{B_{1}} A_{s / 2} u A_{s / 2} \varphi=\int_{B_{1}}|x|^{\alpha} u^{p} \varphi
$$

Definition 1.3. A solution $u \in H^{s}\left(\mathbb{R}^{n}\right) \cap L^{p}\left(B_{1}\right)$ such that $u=0$ in $B_{c}^{1}$ is a weak solution of problem (1.5) if for any $\varphi \in C_{0}^{\infty}\left(B_{1}\right)$ one has

$$
\int_{\mathbb{R}^{n}}(-\Delta)^{s / 2} u(-\Delta)^{s / 2} \varphi=\int_{B_{1}}|x|^{\alpha} u^{p} \varphi
$$

\section{Main results}

The following result is a consequence of a Pohozaev argument.

Theorem 1.4. Let $n \geq 2$ and $s \in(0, n / 2)$. There is no positive smooth solution for problems (1.4) or (1.5) if $p \geq \frac{n+2 s+2 \alpha}{n-2 s}$.

The following theorem is an existence result and is our main result.

Theorem 1.5. Let $n \geq 2, s \in(1 / 2, n / 2)$ and $p<\frac{n+2 s+2 \alpha}{n-2 s}$. There exists a positive weak solution for problems (1.4) and (1.5). 
The following result is a regularity result.

Theorem 1.6. Let $n \geq 2, s \in(1 / 2, n / 2)$ and $p<\frac{n+2 s+2 \alpha}{n-2 s}$. Any weak solution of problems (1.4) or (1.5) for any $1 / 2<s<n / 2$ is $C_{\text {loc }}^{\infty}\left(B_{1}\right)$.

Remark 1.7. Theorem 1.6 is an interior regularity result. The boundary conditions have to be interpreted in the weak sense.

As an application of Theorem 1.5, we have the following result. Let us consider

$$
\left\{\begin{array}{l}
(-\Delta)^{s} u=u^{p} \text { in } B_{1}^{c} \\
u=0 \text { in } B_{1}
\end{array}\right.
$$

One has

Theorem 1.8. Let $n \geq 2, s \in(1 / 2, n / 2)$ and $p>\frac{n+2 s}{n-2 s}$. There exists a positive radial smooth solution $u$ of (1.6).

\section{Proof of Theorem 1.4}

We follow the argument in [9]. Denote by $\mathcal{L}_{s}$ any of our two operators and let $u$ be a smooth solution of (1.4) or (1.5) in $B_{1}$. Consider $u_{\lambda}(x)=u(\lambda x)$ defined in $B_{1}$. Then the following identity clearly holds:

$$
\int_{B_{1}}(x \cdot \nabla u) \mathcal{L}_{s} u d x=\left.\frac{d}{d \lambda}\right|_{\lambda=1} \int_{B_{1}} u_{\lambda} \mathcal{L}_{s} u d x
$$

Note that since $B_{1}$ is star-shaped $u_{\lambda}$ satisfies the desired boundary conditions for both problems. Now, in the case of problem (1.4), we extend the function $u$ by 0 outside of $B_{1}$ and denote it still $u$. As a consequence one has the formula

$$
\int_{B_{1}} u_{\lambda} \mathcal{L}_{s} u d x=\int_{\mathbb{R}^{n}} u_{\lambda} \mathcal{L}_{s} u d x
$$

The function $u$ belongs to the space $\mathcal{H}_{s}\left(B_{1}\right)$ in the case of problem (1.4) and the Standard Sobolev space $H^{s}\left(\mathbb{R}^{n}\right)$ in the case of problem (1.5). Hence it is easy to check by spectral calculus that the integration by parts formula 
holds

$$
\int_{\mathbb{R}^{n}} u_{\lambda} \mathcal{L}_{s} u d x=\int_{\mathbb{R}^{n}} \mathcal{L}_{s / 2} u_{\lambda} \mathcal{L}_{s / 2} u d x
$$

By the change of variables $y=\sqrt{\lambda} x$, one gets

$$
\int_{\mathbb{R}^{n}} u_{\lambda} \mathcal{L}_{s} u d x=\lambda^{\frac{2 s-n}{2}} \int_{\mathbb{R}^{n}} w_{\sqrt{\lambda}} w_{1 / \sqrt{\lambda}} d y
$$

where

$$
w(x)=\mathcal{L}_{s / 2} u
$$

Hence

$$
\int_{\mathbb{R}^{n}}(x \cdot \nabla u) \mathcal{L}_{s} u d x=\frac{2 s-n}{2} \int_{\mathbb{R}^{n}} u \mathcal{L}_{s} u+\left.\frac{1}{2} \frac{d}{d \lambda}\right|_{\lambda=1} I_{\lambda}
$$

where

$$
I_{\lambda}=\int_{\mathbb{R}^{n}} w_{\lambda} w_{1 / \lambda} d y
$$

By Cauchy-Schwarz inequality, one has

$$
\left.\frac{d}{d \lambda}\right|_{\lambda=1} I_{\lambda} \leq 0
$$

Hence one gets

$$
-\int_{\mathbb{R}^{n}}(x \cdot \nabla u) \mathcal{L}_{s} u d x \geq \frac{2 s-n}{2} \int_{\mathbb{R}^{n}} u \mathcal{L}_{s} u .
$$

Using the equation for $u$, one deduces easily the desired result.

\section{Strauss-Ni's Lemma}

The following lemma is due to Cho and Ozawa (see [6]).

Lemma 3.1. Let $n \geq 2$ and $1 / 2<s<n / 2$. Then

$$
\sup _{x \in \mathbb{R}^{n} \backslash\{0\}}|x|^{n / 2-s}|u(x)| \leq C(n, s)\left\|(-\Delta)^{s / 2} u\right\|_{L^{2}}
$$

for any $u$ radially symmetric such that $\left\|(-\Delta)^{s / 2} u\right\|_{L^{2}}$ is finite.

This provides the desired Strauss-Ni's lemma for problem (1.5). Note that the restriction $s>1 / 2$ is due to the convergence of the integrals involved 
in the constant $C(n, s)$. Furthermore, as described in [6], the inequality does not hold for $s \in(0,1 / 2]$ and $s \geq n / 2$.

One now turns to the Strauss-Ni's lemma for problem (1.4). We have

Lemma 3.2. Let $n \geq 2$ and $1 / 2<s<n / 2$. Then

$$
\sup _{x \in B_{1} \backslash\{0\}}|x|^{n / 2-s}|u(x)| \leq C(n, s)\left\|A_{s / 2} u\right\|_{L^{2}}
$$

for any $u$ radially symmetric such that $\left\|A_{s / 2} u\right\|_{L^{2}}$ is finite.

Proof. In the ball, by standard spectral theory, one has the following expression of

$$
u(x)=r^{1-n / 2} \sum_{k} u_{k} \frac{J_{n / 2-1}\left(\sqrt{\lambda_{k}} r\right)}{\beta_{k}}
$$

where

$$
\beta_{k}^{2}=\int_{0}^{1} r^{2-n}\left|J_{n / 2-1}\left(\sqrt{\lambda_{k}} r\right)\right|^{2} r^{n-1} d r=\int_{0}^{1} r\left|J_{n / 2-1}\left(\sqrt{\lambda_{k}} r\right)\right|^{2} d r
$$

and $J_{n / 2-1}(x)$ is the standard Bessel function of order $n / 2-1$.

Hence by Cauchy-Schwarz inequality

$$
|u(x)| \leq r^{1-n / 2}\left(\sum_{k} \lambda_{k}^{s}\left|u_{k}\right|^{2}\right)^{\frac{1}{2}}\left(\sum_{k} \frac{\left|J_{n / 2-1}\left(\sqrt{\lambda_{k}} r\right)\right|^{2}}{\lambda_{k}^{s} \beta_{k}^{2}}\right)^{\frac{1}{2}}
$$

Since we have by spectral theory

$$
\left\|A_{s / 2} u\right\|_{L^{2}}=\left(\sum_{k} \lambda_{k}^{s}\left|u_{k}\right|^{2}\right)^{\frac{1}{2}}
$$

we have to estimate the quantity

$$
\sum_{k} \frac{\left|J_{n / 2-1}\left(\sqrt{\lambda_{k}} r\right)\right|^{2}}{\lambda_{k}^{s} \beta_{k}^{2}}
$$

Setting $\bar{r}=\sqrt{\lambda_{k}} r$, one gets

$$
\beta_{k}^{2}=\frac{1}{\lambda_{k}} \int_{0}^{\sqrt{\lambda_{k}}} \bar{r}\left|J_{n / 2-1}(\bar{r})\right|^{2} d \bar{r} .
$$


The eigenvalues $\sqrt{\lambda_{k}}$ are the zeros of $J_{n / 2-1}(x)$ since, by the boundary condition, we must have $J_{n / 2-1}\left(\sqrt{\lambda_{k}}\right)=0$. It is well-known that one has the following estimate

$$
\lambda_{k} \sim k^{2}
$$

Moreover the following estimate holds (see [1])

$$
J_{n}(x) \sim O(1 / \sqrt{x}) \quad x \rightarrow+\infty
$$

Putting together these two estimates, one has

$$
\beta_{k}^{2} \geq \frac{1}{\lambda_{k}} \int_{0}^{\sqrt{\lambda_{k}}} d \bar{r}
$$

hence

$$
\beta_{k} \sim 1 / \sqrt{k}, \quad k \rightarrow+\infty .
$$

Hence we end up estimating the sum

$$
\sum_{k} \frac{\left|J_{n / 2-1}(k r)\right|^{2}}{k^{2 s-1}}
$$

We split the sum in the following way

$$
\sum_{k}=\sum_{k<<1 / r}+\sum_{k \sim 1 / r}+\sum_{k>>1 / r}=I_{1}+I_{2}+I_{3} .
$$

The sum $I_{2}$ is easily estimated. Indeed, in this regime $J_{n / 2-1}(k r)=O(1)$, hence

$$
I_{2} \leq C r^{2 s-1} \leq C
$$

For $I_{3}$, we use the bound close to $\infty$

$$
J_{n}(x) \leq \frac{C}{\sqrt{x}}
$$

to obtain

$$
I_{2} \leq \frac{C}{r} \sum_{k>>1 / r} \frac{1}{k^{2 s}}
$$

We have

$$
\sum_{k>>1 / r} \frac{1}{k^{2 s}} \sim \int_{1 / r}^{\infty} \frac{1}{x^{2 s}} d x=r^{2 s-1}
$$


Hence,

$$
I_{2} \leq C r^{2 s-2}
$$

For $I_{3}$, we use that (see [1])

$$
J_{n}(x)=O\left(x^{n}\right) x \rightarrow 0 .
$$

Hence we have

$$
\begin{gathered}
I_{3} \leq \sum_{k<<1 / r} \frac{(k r)^{n-2}}{k^{2 s-1}} \leq r^{n-2} \sum_{k<<1 / r} k^{n-1-2 s} \sim r^{n-2} r^{n+2 s}, \\
I_{3} \leq C r^{2 n-2+2 s} \leq C r^{2 s-2} .
\end{gathered}
$$

Therefore, we finally have

$$
\sum_{k} \frac{\left|J_{n / 2-1}(k r)\right|^{2}}{k^{2 s-1}} \leq C\left(1+r^{2 s-2}\right) .
$$

Hence

$$
|u(x)| \leq C r^{1-n / 2}\left(1+r^{s-1}\right)\left\|A_{s / 2} u\right\|_{L^{2}},
$$

which gives the desired bound.

\section{Proof of Theorem 1.5}

The proof follows the ideas of [8]. The proof uses the following mountain pass lemma due to Ambrosetti and Rabinowitz (see [8] and references therein).

Theorem 4.1. Let $E$ be a Banach space and let $J \in C^{1}(E, \mathbb{R})$ satisfy the Palais-Smale condition. Suppose:

- $J(0)=0$ and $J(e)=0$ for some $e \neq 0$ in $E$.

- there exists $\rho \in(0,\|e\|), \alpha>0$ such that $J \geq \alpha$ on $S_{\rho}=\{u \in E:\|u\|=$ $\rho\}$.

Then J has a positive critical value

$$
c=\inf _{h \in \Gamma} \max _{t \in[0,1]} J(h(t)) \geq \alpha>0
$$

where

$$
\Gamma=\{h \in C([0,1], E): h(0)=0, h(1)=e\} .
$$

We now come to the proof of Theorem 1.5. 


\section{The case of the spectral Laplacian}

We consider first the case of Equation (1.4). Let $E$ be the completion of radially symmetric $C_{0}^{\infty}$ functions under the norm

$$
\|u\|_{E}^{2}=\int_{B_{1}}\left|A_{s / 2} u\right|^{2} .
$$

Let

$$
J(u)=\frac{1}{2} \int_{B_{1}}\left|A_{s / 2} u\right|^{2}-\int_{B_{1}}|x|^{\alpha} F(u)
$$

where

$$
F(u)=\int_{0}^{u}|t|^{p} d t .
$$

Clearly, any critical point of $J$ is a weak solution of problem (1.4).

\section{The case of the restricted Laplacian}

We consider now case of Equation (1.5). Let $E$ be the completion of radially symmetric $C_{0}^{\infty}\left(B_{1}\right)$ functions defined on $\mathbb{R}^{n}$ under the norm

$$
\|u\|_{E}^{2}=\left\|(-\Delta)^{s / 2} u\right\|_{L^{2}\left(\mathbb{R}^{n}\right)} .
$$

Notice that functions in $E$ vanish (with all derivatives if necessary) outside of $B_{1}$.

Let

$$
\begin{gathered}
J(u)=\frac{1}{2}\left\|(-\Delta)^{s / 2} u\right\|_{L^{2}\left(\mathbb{R}^{n}\right)}^{2}-\int_{B_{1}}|x|^{\alpha} F(u) . \\
F(u)=\int_{0}^{u}|t|^{p} d t .
\end{gathered}
$$

Clearly, any critical point of $J$ is a weak solution of problem (1.5).

Now the only thing to check to apply Ni's argument is the following compactness lemma (see [8]).

Lemma 4.2. Let $n \geq 1$ and $s \in(1 / 2, n / 2)$. The map $u \rightarrow|x|^{\alpha} u$ from $E$ into $L^{p}\left(B_{1}\right)$ is compact for $p \in\left[1, \tilde{p}_{s}\right)$ where

$$
\tilde{p}_{s}= \begin{cases}\frac{2 n}{n-2 s-2 \alpha} & \text { if } \alpha<\frac{n-2 s}{2}, \\ +\infty & \text { otherwise. }\end{cases}
$$


As in [8], one has

Lemma 4.3. The functional J satisfies the Palais-Smale condition.

The previous two lemmata just need: the compactness of $E$ into $L^{1}\left(B_{1}\right)$ and the Sobolev embedding for $E$. These are proved in the appendix of [2].

\section{Proof of Theorem 1.6}

The case $s=1$ is a well known result. We treat in a unified way both problems and operators.

\subsection{The case $s \in(1 / 2,1)$}

First, by Prop. 4.2 in [12], weak solutions of $(1.4)$ are $L^{\infty}\left(B_{1}\right)$. The very same argument gives $L^{\infty}$ bounds for weak solutions of (1.5). By Prop. 3.2 in [12], solutions are $C^{1, \alpha}$ and hence by a standard bootstrap argument, smooth. For problem (1.5), this is enough to invoke [3], Lemma 4.4 to have the desired result.

Remark 5.1. Regularity results in elliptic theory for integro-differential equations have also been obtained in [9],[4] for this range range of powers.

\subsection{The case $s \in(1, n / 2)$}

The main difficulty now is considering higher order operators. To do so, we follow the strategy of van der Vorst in [13] for the bilaplacian.

Since $u$ is a weak solution of any of the problems (1.4) or (1.5), we have, by the Sobolev embedding that

$$
u \in L^{2 n / n-2 s}\left(B_{1}\right) .
$$

The following lemma can be proved in exactly the same way as Lemma B.2 in [13].

Lemma 5.2. For every $\varepsilon>0$, there are functions $q_{\varepsilon} \in L^{n / 2 s}\left(B_{1}\right), f_{\varepsilon} \in$ $L^{\infty}\left(B_{1}\right)$ and a constant $K_{\varepsilon}$ such that

$$
u^{\frac{n+2 s}{n-2 s}}=q_{\varepsilon}(x) u+f_{\varepsilon}
$$

and

$$
\left\|q_{\varepsilon}\right\|_{L^{n / 2 s}}<\varepsilon \quad\left\|f_{\varepsilon}\right\|_{\infty} \leq K_{\varepsilon}
$$


Now we prove

Lemma 5.3. Let $u$ be a weak solution of any of the problems (1.4) or (1.5). Then $u \in L^{p}\left(B_{1}\right)$ for any $1 \leq p<\infty$.

Proof. Following [13], we rewrite our problems as

$$
u-\mathcal{F}_{\varepsilon} u=h_{\varepsilon}
$$

where $\mathcal{F}_{\varepsilon} u=\left(\mathcal{L}_{s}\right)^{-1}\left(q_{\varepsilon} u\right)$ and $h_{\varepsilon}=\left(\mathcal{L}_{s}\right)^{-1} f_{\varepsilon}$ where $\mathcal{L}_{s}$ is any of our two operators. Now one has (see [2] for $s \in(0,1)$ but the arguments there can be easily adapted )

$$
\left(\mathcal{L}_{s}\right)^{-1} u(x)=\int_{B_{1}} \mathcal{K}_{s}(x, y) u(y) d y
$$

and one has

$$
\left|\mathcal{K}_{s}(x, y)\right| \leq \frac{C}{|x-y|^{n-2 s}} .
$$

Hence using the Hardy-Littlewood-Sobolev inequality one has

$$
\left\|\mathcal{F}_{\varepsilon} u\right\|_{L^{p}} \leq C\left\|q_{\varepsilon} u\right\|_{L^{r}}
$$

with

$$
\frac{1}{r}=\frac{1}{p}+\frac{2 s}{n}
$$

Since $q_{\varepsilon} \in L^{n / 2 s}\left(B_{1}\right)$, one gets by Hölder inequality that

$$
\left\|\mathcal{F}_{\varepsilon} u\right\|_{L^{p}} \leq C\left\|q_{\varepsilon}\right\|_{L^{n / 2 s}}\|u\|_{L^{p}}
$$

The rest of the proof then goes as in [13].

To finish the proof of Theorem 1.6, we invoke the regularity results in [7], Theorem 7.4 to go from $L^{p}$ to a Sobolev-type space and then, by Morrey embeddings, to conclude to the desired regularity. 


\section{Proof of Theorem 1.8}

We will use the following Kelvin transform for $x \in B_{1}$ :

$$
u^{*}(x)=\frac{1}{|x|^{n-2 s}} u\left(\frac{x}{|x|^{2}}\right) .
$$

Denote

$$
x^{*}=\frac{x}{|x|^{2}} \in B_{1}^{c}
$$

and define

$$
u^{*}\left(x^{*}\right)=\frac{1}{\left|x^{*}\right|^{n-2 s}} u\left(\frac{x^{*}}{\left|x^{*}\right|^{2}}\right) .
$$

Notice that $u^{*}\left(x^{*}\right)$ is of course defined on $B_{1}^{c}$. Now by a well-known properties of the Kelvin transform, one has

$$
(-\Delta)^{s} u^{*}\left(x^{*}\right)=\frac{1}{\left|x^{*}\right|^{n+2 s}}\left((-\Delta)^{s} u\right)\left(\frac{x^{*}}{\left|x^{*}\right|^{2}}\right) .
$$

Since $\frac{x^{*}}{\left|x^{*}\right|^{2}} \in B_{1}$, one can use Equation (1.5) to get

$$
(-\Delta)^{s} u^{*}\left(x^{*}\right)=\left|x^{*}\right|^{p(n-2 s)-n-2 s-\alpha} u^{*}\left(x^{*}\right)^{p} .
$$

Take now $\alpha=p(n-2 s)-n-2 s$. Then $\alpha>0$ if and only $p>\frac{n+2 s}{n-2 s}$. Hence we have produced a solution of (1.8) and the Theorem is proved.

Remark 6.1. By the construction of the solution in Theorem 1.8, the solution $u^{*}$ has decay at infinity. More precisely, we have

$$
u^{*}\left(x^{*}\right)=O\left(\frac{1}{\left|x^{*}\right|^{n-2 s}}\right), \quad x^{*} \rightarrow+\infty
$$

\section{Several Open Questions}

We pose several open questions in line with the standard $s=1$ case.

- Is the solution obtained in Theorem 1.5 unique or nondegenerate? This is trivial when $s=1$, by a standard scaling and ODE argument. The case of non local equations is not clear at all.

- It is natural to consider the associated critical or supercritical BahriCoron problem 


$$
\mathcal{L}_{s} u=u^{p} \text { in } \Omega
$$

together with suitable boundary conditions depending on $\mathcal{L}_{s}$, any of our operators, $p \geq \frac{n+2 s}{n-2 s}$ and $\Omega$ exhibits nontrivial topology. We conjecture that there exists a solution to (7.1) when $\Omega$ either has nontrivial topology or $\Omega$ has a spherical hole. For powers $s \in(0,1)$ and when the operator is $\left(-\left.\Delta\right|_{\Omega}\right)^{s}$, the Coron problem has been studied in [10].

\section{Acknowledgements}

The first author would like to warmly thank hospitality of University of British Columbia where part of this work has been carried out.

\section{References}

[1] Milton Abramowitz and Irene A. Stegun, Handbook of mathematical functions with formulas, graphs, and mathematical tables. volume 55 of National Bureau of Standards Applied Mathematics Series. For sale by the Superintendent of Documents, U.S. Government Printing Office, Washington, D.C., 1964.

[2] M. Bonforte, Y. Sire and J.-L. Vazquez, Existence, uniqueness and asymptotic behavior of for fractional porous medium equations on bounded domains. To appear in: DCDS-A, 2015.

[3] Xavier Cabré and Yannick Sire, Nonlinear equations for fractional Laplacians, I: Regularity, maximum principles, and Hamiltonian estimates. Ann. Inst. H. Poincaré Anal. Non Linéaire, 31(1):23-53, 2014.

[4] Luis Caffarelli and Luis Silvestre, Regularity theory for fully nonlinear integro-differential equations. Comm. Pure Appl. Math., 62(5):597-638, 2009.

[5] Antonio Capella, Juan Dávila, Louis Dupaigne and Yannick Sire, Regularity of radial extremal solutions for some non-local semilinear equations. Comm. Partial Differential Equations, 36(8):1353-1384, 2011.

[6] Yonggeun Cho and Tohru Ozawa, Sobolev inequalities with symmetry. Commun. Contemp. Math., 11(3):355-365, 2009. 
[7] Gerd Grubb, Fractional Laplacians on domains, a development of Hörmander's theory of $\mu$-transmission pseudodifferential operators. Adv. Math., 268:478-528, 2015.

[8] Wei Ming Ni, A nonlinear Dirichlet problem on the unit ball and its applications. Indiana Univ. Math. J., 31(6):801-807, 1982.

[9] Xavier Ros-Oton and Joaquim Serra, The Pohozaev identity for the fractional Laplacian. Arch. Ration. Mech. Anal., 213(2):587-628, 2014.

[10] Simone Secchi, Naoki Shioji and Marco Squassina, Coron problem for fractional equations. Differential Integral Equations, 28(1-2):103-118, 2015.

[11] Raffaella Servadei and Enrico Valdinoci, On the spectrum of two different fractional operators. Proc. Roy. Soc. Edinburgh Sect. A, 144(4):831-855, 2014.

[12] Jinggang Tan, Positive solutions for non local elliptic problems. Discrete Contin. Dyn. Syst., 33(2):837-859, 2013.

[13] R. C. A. M. Van der Vorst, Best constant for the embedding of the space $H^{2} \cap H_{0}^{1}(\Omega)$ into $L^{2 N /(N-4)}(\Omega)$. Differential Integral Equations, 6(2):259-276, 1993.

LATP, UMR CNRS 7353, Université AiX-Marseille, France

E-mail address: sire@cmi.univ-mrs.fr

Department of Mathematics, Univ of British Columbia

VAncouver, BC V6T 1Z2 CAnAdA

E-mail address: jcwei@math.ubc.ca

Received April 2, 2014 